Théologiques

Théologiques

\title{
Corps de passions et vérité
} De la réception d'un témoignage de foi

\section{Michel-M. Campbell}

Volume 13, numéro 2, automne 2005

Violence et souffrance rédemptrices

URI : https://id.erudit.org/iderudit/013607ar

DOI : https://doi.org/10.7202/013607ar

Aller au sommaire du numéro

\section{Éditeur(s)}

Faculté de théologie et de sciences des religions, Université de Montréal

ISSN

1188-7109 (imprimé)

1492-1413 (numérique)

Découvrir la revue

Citer cet article

Campbell, M.-M. (2005). Corps de passions et vérité : de la réception d'un témoignage de foi. Théologiques, 13(2), 109-131.

https://doi.org/10.7202/013607ar
Résumé de l'article

La Passion du Christ de Mel Gibson, témoignage de foi public marqué par la violence, a soulevé bien des passions. Cette contribution tente de rendre compte, dans une perspective praxéologique, du jeu complexe des pratiques et des interactions engagées dans la relation particulière du public et des Églises à un film vu et discuté par des dizaines de millions de personnes à travers le monde. L'auteur, qui s'intéresse tout autant à la médiatisation et à la réception du film qu'à sa facture et à son contenu, rend compte d'une partie des discussions, des appréciations (ecclésiales) et des mises en garde (théologiques) par rapport à ce film. Ce qui ressort de ses observations, c'est la puissance de l'industrie de rêves qu'est le cinéma, mais aussi la variété des manières d'aborder le film, dont certaines sont, tout comme le film, marquées par la violence.
Tous droits réservés @ Faculté de théologie et de sciences des religions, Université de Montréal, 2006
Ce document est protégé par la loi sur le droit d'auteur. L'utilisation des services d'Érudit (y compris la reproduction) est assujettie à sa politique d'utilisation que vous pouvez consulter en ligne.

https://apropos.erudit.org/fr/usagers/politique-dutilisation/ 


\section{Corps de passions et vérité De la réception d'un témoignage de foi}

Michel-M. CAMPBELL

Faculté de théologie et de sciences des religions

Université de Montréal

«Tanti corpi, tante passione». Tant de corps. Celui de Jésus mis en images, comme ceux des autres personnages du film La Passion du Christ (2004), mais aussi, les corps concrets de ses acteurs, de ses artisans et particulièrement de Mel Gibson, son réalisateur; corps induits du Jésus des évangiles et de l'imaginaire chrétien; enfin, corps des spectateurs et des critiques du film. Tant de corpus, aussi. Celui du film lui-même, mais également du cortège de toutes ces publicités ou nouvelles qui l'ont annoncé et suivi, sans compter ceux des textes (évangéliques ou autres) auxquels ils réfèrent.

Tant de passions enfin. En négatif comme en positif. Car ce film ne laisse pas indifférent. D'aucuns en souffrent ou craignent qu'il provoque la souffrance; d'autres encore se réjouissent paradoxalement de cette évocation d'une horrible souffrance qu'ils croient rédemptrice. En vrac, quelques exemples. Crainte de certains Juifs que le film provoque un accroissement de l'antisémitisme. Patience de James Caviezel (qui joue le rôle de Jésus) durant le tournage: des heures de maquillage chaque jour, plusieurs blessures graves (il fut, entre autres, foudroyé sur la croix). Difficiles émotions des spectateurs devant la Passion de leur Sauveur ou tout simplement d'un quidam. Inquiétudes ou scandales de théologiens quant à l'orthodoxie ou l'orthopraxie du film. Réjouissances de chrétiens devant un film qui convoque à réfléchir sur le mystère de la foi ou de l'humanité. Sans parler de l'antiaméricanisme ou d'un psychologisme primaire qui ne voit là qu'un autre exemple de masochisme religieux.

Cet essai voudrait rendre compte du jeu complexe de la pratique d'un film (un corpus qui s'impose) comme des pratiques qu'il suscite (sa réception). En l'occurrence, des interactions de ce corpus qu'est La Passion de Mel Gibson, un témoignage de foi, fort de la puissance hollywoodienne, et des différents corps concrets (de personnes) ou métaphoriques (les corpus 
cinématographiques ou littéraires) qui se trouvent mis en jeu par son apparition.

Je procèderai dans une perspective praxéologique. Tout d'abord, une observation: le récit de ma propre appréhension du film. Dans un deuxième temps, une série de réflexions référencées qui permettront de dégager les enjeux des différents protagonistes, leur manière de se situer, pour dégager le (ou les) drame(s) que dessinent les différentes réceptions de ce témoignage de Mel Gibson: une quête de vérité marquée par la violence. Enfin, quelques pistes d'intervention et de prospective. Comme le matériau est fort riche, je choisis la forme du dossier: une écriture brisée, une mosaïque dont les différents éléments hétéroclites sont traités de façons différentes. Non l'écriture linéaire qui enferme dans une perspective, mais un maelström qui tente de rendre la complexité d'un phénomène à qui s'y laisse flotter.

\section{L'observation}

D'entrée de jeu, deux mots sur le sens de cette première partie. Je soumets qu'une des caractéristiques de la théologie pratique est de se situer concrètement. C'est à partir d'un certain nombre de faits observés et partagés avec ses vis-à-vis que s'élabore ce genre de réflexion théologique. Cette observation peut se faire selon les méthodes des sciences humaines (enquête quantitative ou qualitative, observation participante, etc.). En l'occurrence, je présente, sous forme d'un récit, des notes d'un journal de bord qui rendent compte de mon appréhension du film, un phénomène qui peut commencer bien avant son visionnement, voire le dépasser. Il s'agit d'un regard partiel, plus qualitatif que quantitatif ${ }^{1}$ dont le caractère subjectif est avoué. J'essaierai cependant de distinguer, dans mon compte rendu, les faits des réflexions plus personnelles qui seront en italique.

\subsection{Journal de bord. Le film avant le film}

$\left(1^{2}\right)$ L'agence Zenit, le National Catholic Reporter et d'autres journaux annoncent la parution prochaine d'un film sur la Passion, réalisé et financé

1. En date du 11 novembre 2005, Google offre non moins de 1890000 références à la mention «Mel Gibson Passion ».

2. La numérotation relève les principaux faits de ce journal de bord et servira à étayer les affirmations plus ou moins lapidaires des sections suivantes. 
par l'acteur américain Mel Gibson, qui fut la vedette, entre autres, de Braveheart (1995, deux Oscars) et de Patriot (2000). Il y a quelque chose d'étonnant de voir une superstar hollywoodienne, un type plutôt macho, aux personnages violents, s'engager dans un film religieux. J'ai l'intuition qu'il peut s'agir d'un cas de conversion. Ce qui sera confirmé plus tard. Grisé par le succès, Gibson aurait sombré, entre autres, dans la drogue, et c'est au fond du désespoir qu'il aurait retrouvé la foi jusqu'à se faire construire une chapelle dans son domaine où toute sa famille assisterait chaque jour à la messe en latin. Méfiance devant le zèle du converti.

Cascade d'autres informations. En vrac. (2) Le film qui se limite aux douze dernières heures de la vie de Jésus est, semble-t-il, particulièrement violent. (3) Les mouvements de vigilance craignent que ce film n'entraîne une recrudescence de l'antisémitisme en ramenant l'accusation traditionnelle de peuple déicide (dénoncée par Vatican II). Cela attire ma sympathie. (4) Certains voudraient que Gibson modifie son film ou au moins qu'il précise qu'il s'agit de sa propre lecture des événements, ce qu'il refuse. Ceci lui attire ma sympathie. Une ouvre d'art est toujours personnelle. Le droit d'expression de l'artiste comme de l'individu est fondamental. Je ne crois pas que l'ajout de quelques phrases écrites (c'est-à-dire sous un mode non cinématographique) avant ou après le film aurait vraiment infléchi l'impact du visionnement.

(5) On parle aussi du père de Gibson, personnage excentrique, peu sympathique. Révisionniste, il nie le génocide des Juifs par les nazis. Intégriste, il conteste Vatican II et écrit contre Jean XXIII.

Par ailleurs, on précise que (6) par souci de vérité historique, Gibson a choisi de filmer son film en araméen et en latin et qu'il a longtemps résisté à l'emploi de sous-titres. Cette manière de respecter la "vérité historique» laisse songeur. Comme si on faisait la vérité de l'histoire par la simple fidélité matérielle et sans se soucier de la compréhension de cette histoire par le public. Cela me semble cependant cohérent avec un certain catholicisme, où il importe peu que les fidèles comprennent et où c'est la soumission qui compte. Pendant des siècles, on a vécu la messe en latin sans n'y rien comprendre. Au clerc d'expliquer et d'indiquer la voie à suivre.

(7) On apprend que le Pape a vu le film et qu'il a déclaré à ses proches que "ça s'était bien passé comme ça ». Une déclaration qui a fait sursauter des vaticanologues. On s'étonnait du caractère absolument inédit de l'affaire: le Pape qui sanctionne positivement un film, du jamais vu! On a aussi critiqué le manque de clarté du bureau de presse du Saint-Siège qui 
chercha dans un premier temps à nier la nouvelle. Mais le mal ou le bien était fait. Par ailleurs, le Saint-Siège a continué à faire plus ou moins implicitement la promotion du film: Zenit, une agence informatique qui diffuse des nouvelles du Vatican, a multiplié les reportages (témoignages, lettres pastorales d'évêques, annonce de la deuxième audience de James Caviezel, qui joue le rôle de Jésus, etc.).

Enfin, (8) si les diffuseurs français semblaient réticents à présenter un film d'une telle violence, (9) ailleurs les Églises elles-mêmes prenaient sur elles d'en assurer la promotion et réservaient à l'avance des salles de cinéma entières pour y transporter leurs fidèles.

Tout cela ne m'inspire guère. Je me méfie du genre de cinéma américain hyperindividualiste qui cultive le culte de la personnalité du héros au détriment des autres membres de la communauté. Tous ces discours pour ou contre ce film, comme la date de sa sortie (le mercredi des Cendres), me semblent constituer une excellente campagne de promotion. Ce ne sont pas (10) les 25 millions investis par Gibson qui vont m'émouvoir. Il en a gagné autant pour son dernier rôle et il va les recouvrer largement dès les premières semaines de projection en salle. Je n'ai que faire d'une vision du monde où se croisent celles du président Bush et de Jean-Paul II. Comme "consommer, c'est voter", j'ai décidé de ne pas y consacrer 11 \$.

\subsection{Le visionnement}

Un filleul se dit intéressé à aller voir ce film avec moi pour en discuter. Comment résister à une telle invitation? Je dois cependant avouer (11) avoir vu le film dans une posture particulière. Habituellement, j'essaie de me laisser porter par le film, sans esprit critique, pour bien m'en imbiber et pouvoir asseoir, plus tard, mon interprétation sur le plus d'éléments sémantiques possible. J'ai abordé ce film d'une manière que je dénonce souvent: en maître suspicieux, prêt à corriger. J'avais deux critères: premièrement, la prétention à la vérité de Gibson (entérinée par Jean-Paul II) ou du moins au respect des textes évangéliques; deuxièmement, ma propre prétention à ne trouver là qu'une version religieuse du genre superhéros américain qui me semble en opposition avec la théologie du corps $d u$ Christ, proposée par le Jésus des évangiles.

D'énormes écarts entre le film et les textes évangéliques sont vite apparus. Par exemple, (12) où trouve-t-on que Jésus ait été battu et torturé avant d'arriver au Sanhédrin? Qu'il ait parlé latin ? Ou encore, cette scène où Marie, mère de Jésus, et Marie de Magdala reçoivent des serviettes de 
la femme de Pilate pour recueillir sur le sol le sang de Jésus? Sans parler de la double flagellation? La promesse de fidélité historique de Gibson ne tient pas. Elle était pipée comme pour la plupart des reconstitutions historiques. Cette prétention de Gibson, comme du placet papal qui s'ensuivit, à l'adéquation des images avec la réalité doit se situer à un autre niveau plus général, plus théologique, dans le genre de mon second critère.

Mais là encore, le film ne passe pas. (13) Le Jésus des évangiles m'apparaît comme antinarcissique. Il n'exalte jamais son corps individuel; au contraire, il évide son individualité pour exalter le corps des autres. Que de fois, à l'occasion des miracles, il récuse sa puissance et déclare que c'est la foi de l'autre qui a sauvé le miraculé. Il dénonce les querelles de préséance de ceux qui veulent siéger auprès de lui. Son corps est ailleurs. Il s'identifie aux corps de ceux qui sont dans le besoin (Mt 25). Ce n'est pas le corps $d u$ maître devant lequel on s'incline, mais, paradoxe, celui du maître qui lave les pieds de ses disciples pour les amener à faire de même. Et dans les récits de la Cène, il s'identifie avec les réalités les plus quotidiennes de sa culture, le pain et le vin.

Or, (14) le Jésus de Gibson me semble beaucoup plus près des superhéros américains. Cet homme a une force, une capacité de souffrir surbumaines qui le séparent de ceux qui l'entourent. Il n'a pas un corps de la même nature que celui des autres. D'ailleurs, (15) au moment où il prend la croix, Marie se demande jusqu'où ira sa patience: "Mon fils, quand, où, comment décideras-tu de te délivrer de cela? " Comme s'il pouvait à un moment donné, en vertu de sa puissance divine, interrompre son tourment. Cet homme n'est pas vraiment humain, avec les limites d'un corps humain. Les torturés des prisons irakiennes, pré- ou post-invasion étatsunienne, ne peuvent décider du quand, du où, du comment de leur délivrance. Le Jésus de Gibson joue à l'humain en occultant une réserve qui pourrait invalider l'acharnement qu'on manifeste contre lui. (16) Cette phrase de Marie, absente des évangiles, a pour moi un relent de docétisme (hérésie selon laquelle le corps de Jésus n'est qu'une apparence). Me voici, inquisiteur!

(17) À ceux qui disent que ce personnage fait penser à toutes ces victimes de procès véreux, de torture ou de lynchages, je répondrais qu'il y a là généreuse projection de leur part. J'ai moi-même été tenté de la faire, mais ce manque de limite, cette puissance cachée du Jésus du film, dissociait son corps des autres corps de victimes.

Lors de la discussion, (18) mon filleul n'est guère revenu sur le film, mais il a posé une question intéressante sur la difficulté d'accepter la 
prétention du Jésus des évangiles à être le personnage central de l'histoire quand on a maintenant conscience de la valeur des autres religions, voire de l'immensité du cosmos. Ce qui a entraîné (19) une réflexion sur les genres littéraires.

\subsection{Après le visionnement}

Quelques incidents. Le premier, particulièrement articulé. J'aborde une conversation à bâtons rompus entre un spécialiste européen de la violence et des collègues des sciences religieuses (dont des exégètes) où l'on discute fermement du film. Je comprends que (20) les objections reprenaient, en grande partie, l'argument de la violence extrême qui s'y manifeste. Ce spécialiste défend le film. D'après lui, (21) il ne faut pas, comme notre époque a tendance à le faire, refouler la violence réelle et il rappelle des exemples de violence extrême dans la longue tradition iconographique chrétienne (entre autres, le triptyque de Grünewald). Il n'y a donc pas là raison d'interdire le film.

Je lui rétorque qu'il ne s'agit pas pour nous de vouloir censurer ce film. (À ce sujet, on ne m'a rapporté que la question d'un collègue (22) qui demandait jusqu'où on pouvait tolérer ce genre de production.) Je précise que notre critique porte sur la prétention à la fidélité aux évangiles et que, par exemple, Gibson aurait dû reconnaître les emprunts faits aux révélations d'Anne-Catherine Emmerich. Le spécialiste s'est alors montré sensible à notre position en ajoutant (23) avoir pris, jusque-là, une perspective largement européenne.

Quelques autres incidents, en vrac. (24) Une étudiante me dit qu'elle ira sans doute voir le film, mais qu'elle a peur de voir Jésus souffrir. Sentiment bien partagé. (25) D'autres étudiantes, sensibilisées à une christologie moderne et engagées socialement, disent ne pas retrouver le Christ dans ce film. (26) Zenit présente la méditation d'une religieuse à la suite du film. Elle y déclare d'entrée de jeu qu'étant donné qu'elle est une femme, elle ne pouvait s'identifier à Jésus et devait s'identifier à Marie, sa mère. Je ne suis pas allé plus loin, découragé par ces relents d'une certaine nouvelle théologie romaine: de la logique de l'accès du sacerdoce aux femmes, découlerait une interdiction de l'imitation de Jésus Christ! Par ailleurs, on me rapporte (27) la réflexion naïve d'une bonne dame qui, après une discussion plus ou moins savante sur le film, se contentait de déclarer sa joie devant la ressemblance du personnage à Jésus. Pieuse pornographie (au sens technique, 
représentation complaisante d'un corps, comme celui de la prostitution). Phantasme naïf. Qui peut dire à quoi ressemblait Jésus?

Apprenant qu'on discuterait de ce film à la présente session (automne 2004) de théologie pratique, (28) un collègue suggère d'y substituer un thème plus fondamental. Pour lui, un film reste une réalité éphémère. Le film a sans doute été vu par quelques dizaines de millions de spectateurs à travers le monde et c'est loin d'être fini. Ainsi, le 12 septembre 2004, Zenit annonçait sur son site Web que, dès la première semaine de la distribution, neuf millions de DVD et vidéocassettes de La Passion avaient été vendus; un record absolu selon la 20th Century Fox Home Entertainment. Il faudra aussi compter le nombre de reprises à la télévision du film, année après année, à l'occasion de Pâques.

Un autre collègue à qui j'évoque le projet de la présente publication me déclare (29) qu'on ne peut que dénoncer un tel film.

(30) La Commission doctrinale de la Conférence des évêques de France publie le 31 mars 2004 une Note doctrinale sur La Passion du Christ, film de Mel Gibson (voir Vallin 2004). On y lit que "ce témoignage d'un Chrétien sincère doit être pourtant soumis, plus que d'autres, à la vigilance des pasteurs de l'Église ». (31) Un évêque français, moins prudent, précisait dans sa propre note doctrinale qu'il s'agissait d'un témoignage de foi

Après une affirmation de principe — «Aucun Chrétien n'est assuré de produire un témoignage chimiquement pur» -, (32) on y déclare qu'il s'agit d' "un film efficace, dont la prouesse technique, dans le genre d'un Gladiator, rencontrera les goûts du public », car il «correspond à des attentes très puissantes du public, mais très suspectes ». La note doctrinale ajoute:

... l'option esthétique la plus périlleuse de ce film réside dans le parti pris d'isoler la passion [sic] de la prédication de Jésus, d'un premier côté, et des récits sur le Ressuscité, d'un autre côté. La littéralité de la violence revêt dans l'isolement des scènes de la Passion, une brutalité presque absurde, à peine illustrée par des retours en arrière sur la vie publique du Christ, et les trois ans de sa prédication. [...] il est regrettable que soient occultés tous les motifs complexes qui ont peu à peu fait monter à la fois l'adhésion des foules à Jésus, et aussi la controverse sur sa personne, ses intentions, son mystère. [(33)] [...] Or, Jésus a choqué [...], a provoqué des questions légitimes parmi les Juifs ses frères. Les réponses qu'il a apportées n'étaient pas mécaniquement convaincantes et supposaient qu'[on] [...] s'en remett[e] à son autorité inouie par un acte de foi, renouvelé à la racine. [...] 
Le spectateur moins averti est exposé au risque de ne comprendre dans ces deux heures d'horrible lynchage qu'une espèce d'événement erratique, un déchaînement de violence furieuse, démente, incompréhensible en tout. Pire: [(34)] il n'est pas exclu que l'attitude de Jésus soit interprétée selon les catégories ambiantes du système paradoxal de la non-violence, ou même de la structuration névrotique de la corrélation sado-masochiste. [...]

De l'autre côté, [(35)] la résurrection est ici montrée, contre l'esprit des évangiles, comme un événement en solitaire et perceptible de soi, antérieur à la logique de rencontre et de témoignage des apparitions. [...]

Cette option d'isolement de la Passion conduit à une autre équivoque théologique de grande portée: [(36)] le péché du monde, et en face de lui, l'intention de salut et de pardon qui dirige l'existence du Fils de Dieu venu parmi les hommes, ne sont pas dans la nécessité, là encore toute mécanique, de se négocier au prix du sang. Comme si Dieu, en sa Toute Puissance, était de toute éternité soumis à une règle souveraine qui l'oblige et le contraigne, lui aussi, le Dieu infiniment libre: l'injustice des hommes ne pourrait être compensée, corrigée, guérie que par la justice de Dieu le Père mais au prix des souffrances et de la mort du Fils.

«Ma vie, nul ne la prend mais c'est moi qui la donne», dit au contraire Jésus. «Nul n'a pris la vie » du Christ, encore moins une espèce de règle abstraite de compensation. [(37)] C'est au contraire l'amour de Dieu et sa miséricorde qui ont représenté devant nous, pour nous convertir le cœur, la logique tueuse du péché. Logique à l'œuvre dans l'histoire de ce monde et entre nous, logique qui s'en prend même à l'Homme juste, à l'Homme bon, à l'Innocent. Alors, rejoint jusqu'à l'intime par la logique du péché de ce monde - c'est l'agonie — le Christ Jésus va pourtant vivre et même exposer en sa mort l'extrême de son amour: sa totale liberté d'aimer va dominer la nécessité mécanique du péché.

Il ne faut pas dire que notre cinéaste soit étranger à ce mystère de la miséricorde divine. Mais la nécessité du sang réparateur est ici en grand péril de masquer la décision filiale de l'amour. Les raisons de la miséricorde ont eu chez lui moins de place pour s'expliquer que les déraisons, et même les démences du péché. [...]

La croix que l'Église célèbre est celle que Jésus a demandé aux disciples de prendre sur leurs épaules pour le suivre et l'imiter. [(38)] Or, le film de Gibson montre la croix inimitable, repoussante, absurde. Il semble pourtant qu'on puisse croire, avec l'Évangile de Jean, que la Mère de Dieu et «le disciple bien aimé » devant Jésus crucifié aient su dépasser dans un acte de foi abyssal l'extrême de la douleur, et qu'ils aient reçu alors d'y contempler quelque 
chose de l'extrême de l'amour. C'était cet amour seul qu'il faudrait imiter. Avant nous et pour toute l'Église, l'une et l'autre commençaient peut-être à éprouver ce que les témoins du jour de Pâques allaient communiquer à tous les Chrétiens, le mystère résumé en ce cri qu'on voudrait lancer à l'adresse de Mel Gibson: "Cette croix, nous l'avons trouvée belle!». François d'Assise, Jean de la Croix, Maximilien Kolbe n'ont pas embrassé une autre croix que celle de l'amour extrême, la croix glorieuse, la croix de vie.

[(39)] À voir ce film, on se demanderait presque si les seuls disciples authentiques du Jésus de M. Gibson ne seraient pas ces candidats exotiques à l'imitation du Crucifié que la télévision nous montre chaque Vendredi Saint, entrant dans une mimétique exacte des tourments du Christ (coups, plaies, clous), mais si extérieure aux profondeurs de l'amour [...].

On doit s'interdire d'instruire un procès d'intention contre l'auteur de ce film sur le sujet de l'antisémitisme. [(40)] Mais il demeure vrai qu'objectivement, le parti qu'il a pris de ne rien montrer de la violence des controverses entre Jésus et les Pharisiens, les scribes, les chefs de prêtres, aboutit à cet effet de mutilation mécanique: les Juifs du Sanhédrin sont ici largement privés de l'expression des motifs, reçus de la Révélation elle-même, qu'ils avaient eu d'être au moins surpris, heurtés, contredits, par la prédication du Rabbi de Nazareth. On les prend à l'heure du procès comme à l'heure d'une colère démente, invincible et sournoise. C'est au moins mentir à la dramatique intégrale des évangiles. (Vallin 2004)

Gibson vient de passer au tribunal d'un inquisiteur bienveillant, d'un bon maître qui a corrigé le film, en montrant ses lacunes ou ses dangers. On peut s'interroger sur l'efficacité de tels propos. Certes tout cela a du sens et valait la peine d'être souligné. En même temps, ce genre de critique qui, en toute logique, demanderait d'amender le film, risque de s'avérer lettre morte. Qui l'a lue, quel public a-t-elle rejoint? Sans doute, une goutte d'eau dans l'océan des spectateurs du film.

Par ailleurs, (41) la théologienne Lise Baroni et le psychanalyste Michel Dansereau offrent une défense enthousiaste du film, défense qu'ils intitulent, paradoxalement, La Passion du Christ: un film intolérable. Ne nous trompons pas de cible. D'entrée de jeu, ils constatent "l'effervescence des réactions" et se demandent "si nous sommes en train de sombrer dans la confusion totale ou, au contraire, d'émerger à un surplus de compréhension et de sens ». Pour leur part, ils voient là « une occasion exceptionnelle de revoir nos positions, surtout si celles-ci sont murées dans des certitudes intouchables. [Car] la fermeture idéologique n'appartient pas qu'aux autres ». 
Ils notent que "le christianisme a cru bon de retenir au moins quatre versions du même Évangile [et que] celles-ci ne présentent pas toujours entre elles une cohérence implacable sur le plan historique". Ils "comprenn[ent] qu'on fasse une lecture exégétique pure et dure », mais ils affirment s'être laissés «rejoindre par d'autres dimensions", en particulier le point de vue des artistes. "Surtout quand il leur arrive de faire œuvre de véritables théologiens sans nécessairement le vouloir ou même le savoir. Ce fut le cas du [(42)] Jésus de Montréal de Denis Arcand qui re-lit d'une façon exceptionnelle, la Passion de Jésus dans le contexte du génie et de la folie qu'offre le monde contemporain ». (43) Ils citent alors toute une série de visions valables de la Passion en peinture (Rembrandt, Michel-Ange, Rouault, Dali), en musique (Bach, Haendel, «sans parler d'un subtil nocturne de Schubert») et, plus tard, en cinéma (Pasolini, Zeffirelli, Connor, Arcand, Scorsese). (44) À l'instar de Louis Lavelle, ils évoquent une rivalité entre philosophie (ou discours du savoir) et poésie (ou œuvre d'art). La première "oblige à penser le monde qu'[elle] ne peut montrer alors que la «réalité que présente l'artiste peut rendre l'irréel plus réel que le réel banalisé de nos jours d'inconscience!»

Pour eux, on a du mal à aimer ce film. Le vivre est une véritable épreuve. Ils reconnaissent «des accrocs notables au plan exégétique ", des libertés avec l'histoire, mais ils refusent «de se laisser enneiger dans leurs certitudes scientifiques». Ils acceptent de se laisser toucher par «la lecture» de ce croyant, converti, certes conservateur («et puis après»), avec la violence qui a marqué sa réception de la Passion. Ils osent "affirmer que Gibson n'a rien exagéré de la souffrance, physique certes, mais aussi de la solitude, de rejet, d'abandon que doit endurer toute personne condamnée alors qu'elle se sait innocente ».

Ils marquent leur désaccord avec (45) ceux pour qui ce film n'a rien à voir avec le christianisme:

Rien de plus faux: cette Passion est au cœur du christianisme et la gommer ne peut qu'occulter aussi celles que vivent encore tant d'êtres humains [...]. Comprendre cela, le recevoir comme une réalité éminemment actuelle [le film était sur les écrans au moment où se remémorait la Passion des Rwandais] est, alors là, oui, quelque chose d'intolérable. Mais la lucidité et la mémoire vive sont des voies qui contribuent à trouver la sortie vers la vie.

Bref, aussi problématique qu'il puisse apparaître à la lumière des sciences, [ce] film, prend tout son sens lorsqu'on en fait une lecture contextualisée (nous ne disons pas intellectualisée) de type symbolique, voire mystique. 
C'est peut-être même cette lecture qui lui rend le mieux justice puisqu'il s'agit d'une œuvre artistique.

Ils liront donc le film avec un principe qu'ils partagent avec (46) l'exégète Jean Duhaime (qui a pourtant écrit qu'il trouvait le film «dégoûtant»): «la fidélité à l'Évangile [... consiste] en une rencontre dialogique avec un texte dont on s'approprie le sens dans un autre contexte. » (2004) Ils entreprennent de relire le film comme une réécriture de l'Évangile par une série de paragraphes apologétiques dans lesquels ils reprennent ses différents personnages (figures du démon, des enfants, de Marie, Simon de Cyrène, Pierre et Véronique) et ses thèmes, ou à partir des éléments des critiques (violence, masochisme, dolorisme, résurrection, culpabilité). Chacun de ces éléments est traité dans un va-et-vient entre l'Évangile et le monde d'aujourd'hui. Parfois nommément en opposition avec un critique. Il serait trop long de reprendre chaque cas. Je me contente de retenir quelques citations de leur réaction à l'accusation de masochisme pathologique.

Pour eux, (47) "Jésus ne prend aucun plaisir vicieux à tendre l'autre joue». Ils retiennent cependant la scène du «baiser [de Jésus] à la croix sur laquelle il sera cloué [...], un symbole du film qui ne se trouve pas dans les récits historiques mais [qui] se retrouve dans les textes mystiques». Ils ajoutent:

Les pères de l'Église n'ont pas hésité à voir dans le sacrifice de Jésus un hieros gamos [mariage divin] avec la mère. Par sa mort, il retourne dans le sein de sa mère terre et rachète le mal où échoua Adam comme CEdipe. [(48)] Saint Augustin qui se connaissait en matière de relations charnelles amoureuses et n'avait pas peur des mots, affirme: "Tel un fiancé Jésus sortit de sa chambre et s'avança prédisant son mariage dans la plaine du monde. Il s'avança jusqu'au lit de la croix et en y montant, il confirma son union. Et quand il sentit les lourds soupirs de la créature, en un pieux abandon, il s'est sacrifié pour racheter son épouse [l’Église], et il s'est fiancé à la femme pour l'éternité ». Le texte latin est très vigoureux et affirme sans aucune pruderie: Et copulavit sibi perpetuo iure matronam!

On est loin des perspectives historiques étroites ou des corrections $d u$ maître. On se trouve avec des gens choqués par la souffrance intolérable, mais qui l'assument, à bras-le-corps, dans la lucidité de leur culture et de leur foi. Le film est reconnu dans son genre comme une œuvre d'art et situé dans la salle Passion d'un Musée imaginaire pluridisciplinaire. On l'aborde non comme un discours académique, mais comme une ouvre qui touche et qui parle, d'hier comme d'aujourd'bui, de l'universelle question de la 
souffrance. La réponse s'inscrit dans la tradition chrétienne. Une tradition sans doute fort loin des clercs qui ont réservé des autobus pour remplir des salles de projection de la Passion. Celle du baiser de la Croix. Ce baiser, dans le langage bien incarné d'Augustin - ce lit, ces lourds soupirs de la créature qui précèdent le pieux abandon, enfin, la copulation de droit éternel - marque l'assomption de la souffrance dans la force, la grandeur et la profondeur où se conjuguent les mystères de l'Amour de Dieu et de l'Éros. Gibson n'y a sans doute pas pensé. Ce n'était pas son rôle. Reste que son film l'a donné à penser pour un couple de lecteurs dont la réécriture du film a du sens.

\section{Des pointes d'observation}

De ce matériel plus ou moins hétéroclite, qui illustre cependant la complexité des pratiques autour de la réception d'un film ou d'un témoignage de foi public, il est possible de tirer un certain nombre de pointes qui permettront d'élaborer une problématique. Je les présente sous forme de propositions, tout en renvoyant partiellement, en note, à la numérotation des faits effectuée plus haut.

A) Ce genre de film ou de témoignage ne se présente pas comme un pur objet scientifique. Avant même qu'on le lise en lui-même, il nous arrive par la rumeur, elle-même chargée d'une série d'éléments connexes qui lui sont parfois étrangers: par exemple, les notes biographiques, plus ou moins positives, au sujet des Gibson, père et fils ${ }^{3}$.

B) On ne le reçoit pas, non plus, de façon objective ou neutre. Chacun le situe dans sa propre perspective. Chacun cadre le film à sa façon, fait du cinéma à sa manière à propos de ce film, quitte à y introduire des éléments ou des personnages qui lui sont plus ou moins étrangers. Cela va de la peur d'une recrudescence de l'antisémitisme au désir de retrouver l'image du Jésus de sa foi en passant par le souci du respect du texte évangélique ou le diagnostic d'un refoulement de la violence par notre société4.

C) En fait, sauf exception, le corpus du film dans son ensemble apparaît très peu dans les différentes appréciations qu'on en fait. S'il y a eu

3. Pour étayer cette proposition, voir par exemple les numéros 1, 3, 4 et 5 de ma numérotation. Je remercie M. Philippe St-Germain pour sa collaboration non seulement dans l'édition de cet article, mais aussi pour son travail particulier de référence dans cette section. Ces renvois sont en notes de bas de page pour alléger le corps du texte.

4. Voir numéros 6, 7, 9, 11, 17, 19, 21, 23, 25, 26, 35, 41 et bien d'autres encore. 
procès, on a vite escamoté l'objet du litige (la Passion telle que filmée) pour des perspectives propres à chacun (retrouver le visage de Jésus ou encore, à l'autre extrême, épouser l'insupportable souffrance) $)^{5}$.

D) Pourtant, les enjeux sont de taille. Il s'agit de foi et de vérité, de la pertinence d'un témoignage ou du danger d'un contre-témoignage puissant, du problème de l'assomption de la souffrance, voire du cœur de l'économie humaine et chrétienne ${ }^{6}$.

E) Pis, le film est objet de scandale ${ }^{7}$. Cette représentation de la Passion renforce le clivage des chrétiens: entre fondamentalistes et modernistes, entre simples croyants et intellectuels. De part et d'autre, on juge, on n'écoute pas. Le mépris rôde.

F) Enfin, dans la perspective plus particulière de la pratique théologique, ce phénomène marque l'inégalité des forces en présence, de l'efficacité des discours théologiques versus la machine industrielle du cinéma hollywoodien, en collusion avec des pouvoirs religieux ${ }^{8}$.

\section{Le drame de fond}

Une quête de vérité marquée par la violence. L'une et l'autre constituent la trame du long récit de mon journal de bord. De la violence de l'intégrisme de Gibson père à la rigidité du catholicisme de son fils converti, en passant par la violence de la déchéance de ce dernier au moment de sa conversion (quête de vérité). Des prétentions à la vérité historique du réalisateur (quête de vérité) au caractère insupportable de la majorité des images de son film. Violence appréhendée d'antisémitisme. Dénonciation, au nom de la vérité scientifique ou dogmatique, des violences que fait subir Gibson aux textes évangéliques, voire à la juste compréhension de l'économie de la croix. Violence faite à la vérité du film lui-même, à son corpus, la plupart du temps largement ignoré par des jugements à l'emporte-pièce quand on ne désire pas tout simplement censurer le film.

À un niveau plus fondamental, violence de la vérité, de la réalité même de l'insupportable souffrance que l'on cherche spontanément à éviter, quitte

5. Voir numéros $18,25,27$, etc.

6. Voir numéros $12,13,38,40$, etc.

7. Voir numéros 22, 30, 45, 46, etc.

8. Voir numéros 10, 14, 15, 32, 39, etc. Les lecteurs auront compris les retours des pointes sur les éléments observés. Pour cette raison et pour des raisons d'économie, nous arrêtons ici d'expliciter le retour à l'observation. 
à «se tromper de cible». Violence du paradoxe de la proposition chrétienne et/ou psychanalytique (pour qui n'a pas accès à ces sommets) que c'est en embrassant la croix, en chérissant la souffrance comme le jeune marié sa nouvelle épouse, que se trouve le salut.

Violence faite au corps du Christ, à la communauté ecclésiale, quand chacun, sous prétexte de sa propre orthodoxie, nie toute valeur au discours d'autres chrétiens et se cantonne dans sa solitude. Violence faite au corps du Christ, l'humanité souffrante, torturée, encore aujourd'hui crucifiée, violence qu'un tel film peut, selon les interprétations, condamner à la logique du sang réparateur ou encore amener à compatir à tout corps «despised and rejected». Sans compter, en mode mineur, la violence de la force médiatique et des pouvoirs institués, contre la parole critique. Ou encore, celle faite à cette malheureuse religieuse qui se croit interdite, par une théologie des mieux autorisées, de s'identifier à Jésus-Christ pour une question de genre.

Comment défaire ces nœuds mortifères? Tout d'abord en tentant de les comprendre, ce qui est le rôle de l'instance d'interprétation en praxéologie.

\section{L'interprétation théologique}

Il s'agit maintenant de se doter de référents personnels ou littéraires qui permettront de structurer du sens dans le phénomène observé. L'universitaire chrétien les trouvera dans sa double tradition (modernité et héritage judéo-chrétien) et il essaiera d'en bricoler une intégration. Nous en proposons, de façon très schématique, quatre: une réflexion personnelle sur l'imaginaire industriel qu'est le cinéma versus la parole; la relecture critique d'une typologie de Michel Serres des approches d'un texte; l'évocation du passage du monde du signifié à celui du signifiant; enfin, une intuition sur la nécessité d'une pluralité pour dire DieuE.

\subsection{Industrie de rêves et passion de paroles}

\subsubsection{Thèse: le cinéma comme industrie de rêves, c'est-à-dire pornographique}

Non plus le rêve qui surgit, qui advient, qui surprend dans le dé-contrôle du sommeil. Mais un rêve marketé, préfabriqué pour séduire le consommateur. Pas d'inédit, de la complaisance. Hollywood producteur de porno. Version hard: répétitions de variations sur la scène primitive pour stimuler 
ceux qui peinent à la revivre sans image. Version soft: histoire dont la fin est contrôlée par des vérifications auprès d'un public test. On choisit celle qui aura le plus d'attrait commercial. Du même au même: le consommateur roi, tout-puissant, qui peut continuer de dormir, car il contrôle même ses rêves. On est loin du cinéma d'auteur qui provoque par ses thèmes et son style personnel, qui produit du symbole, qui déstabilise, fatigue, «donne à penser ». Encore faudrait-il prendre le temps de voir si l'œuvre de Gibson n'est pas aussi, malgré son genre, parole d'auteur.

\subsubsection{Parenthèse historique sur la récupération de la Passion par l'industrie cinématographique}

Défi des débuts du cinéma: amener le public dans la salle et le fidéliser. On offre un ustensile ou un plat chaque semaine: les amoureux, public cible, reviendront pour compléter le service de vaisselle ou la coutellerie. On invente la série, court métrage qui se termine par un point de suspension... auquel le spectateur ne pourra échapper qu'en revenant la semaine suivante. Dans la même veine, on invente le film religieux (histoires bibliques, de conversions, vies de saints) qui attirera les chrétiens dont la théologie d'incarnation est iconophile. Cependant, les industriels se font prudents? Représenter la Passion, par exemple, est aventure périlleuse. Il y a la question Véronique (de vera icôna). Question d'orthodoxie, quête de vérité. Pathé s'en tirera en filmant la Passion ${ }^{10}$ d'Oberammergau, qui a le nibil obstat romain.

\subsubsection{La question de l'orthodoxie}

Cette question de l'orthodoxie reste entière alors même que les Églises ne menacent plus l'industrie cinématographique. Pasolini, par exemple, qui n'avait rien d'un conformiste, insistait pour dire que sa Passion ${ }^{11}$ se distinguait de ses autres films et qu'il avait travaillé avec des théologiens sérieux $^{12}$. La question du marketing reste tout aussi présente. Quel coup

9. Des travaux d'historiens du cinéma de l'Université de Montréal ont alimenté les dernières phrases de ce paragraphe, notamment Gaudreault 1992; Raynauld 1992.

10. Le film en question est La Passion de Notre-Seigneur Jésus Christ (1902), coréalisé par L. Nonguet et F. Zecca. Le film a été retravaillé et a pris sa forme finale en 1905.

11. L'Évangile selon saint Matthieu (1964).

12. Conviction énoncée par le cinéaste lors d'un entretien avec l'auteur de ces lignes. 
d'avoir une déclaration papale (même officieuse) sur l'adéquation du film et de la réalité!

\subsubsection{Le questionnement iconoclaste}

Reste le questionnement iconoclaste, qui s'inscrit du Lévitique à la théologie de la libération en passant par saint Bernard et Luther. Un nibil obstat ou l'organisation de projections par des pasteurs annulent-ils le fait que le film, comme l'idole, est fabrication humaine et non réalité. Comme le rêve fabriqué qui n'est pas un vrai rêve. Et que le spectateur, comme le rêveur, s'il veut sortir de sa passivité, passer à la parole vive, devra subir l'épreuve du procès, de la passion, des interprétations.

\subsection{La typologie des approches du corpus}

Michel Serres (1974, 221-237) énonce quatre manières d'aborder un texte ou un corpus.

\subsubsection{La doxologie}

Par la doxologie on cherche à retrouver les antécédents du texte (sa généalogie, son archéologie). Comme dans les références des marges de certaines éditions de la Bible qui renvoient à des textes antérieurs (la métaphore est de moi). Dans ce cas, le texte approché ( $\mathrm{T}$ ) trouve sens dans d'autres textes antérieurs (Ts), ce qui donnerait $\mathrm{Ts} / \mathrm{T}$ (le mathème est de moi, comme tous ceux qui suivent). Dans mon journal de bord, on retrouve cette approche au sens strict chez les biblistes ou chez celui qui évoque les révélations d'Anne-Catherine Emmerich. On la retrouve aussi de façon fort généreuse dans le texte de Baroni et Dansereau qui renvoie à toute une série de Passions, musicales ou autres. De façon analogique, c'est une préoccupation constante de Gibson, des biblistes, ou des ecclésiastiques.

\subsubsection{Le commentaire}

Le commentaire présente l'approche du commentateur, de celui qui veut faciliter la saisie du texte par quelqu'un d'autre. Il résume les passages facilement compréhensibles et explicite ce qui apparaît trop concis. Dans ce cas, le texte approché $(\mathrm{T})$ trouve sens par un sujet $(\mathrm{S})$ pour d'autres sujets $(\mathrm{Ss})$, ce qui donnerait $\mathrm{S} / \mathrm{T}(f)$ Ss. Dans mon journal de bord, on retrouve cette 
approche chez le spécialiste de la violence et chez Baroni et Dansereau. Dans un cas comme dans l'autre, on s'intéresse non seulement au corpus du film, mais aussi à notre société qui risque de "se tromper de cible».

Notons dans ce cas l'apparition de sujets qui peuvent par leurs besoins (Ss) ou par leur culture (S) enrichir l'approche du texte. Ce qui s'exprime de façon remarquable chez Baroni et Dansereau.

\subsubsection{L'interprétation}

L'interprétation représente l'approche de celui qui considère un corpus à partir d'un système ou d'une théorie, puisés dans une série de textes qu'il privilégie (TPs) pour y trouver une confirmation ou des dérapages: par exemple, lire un texte dans une perspective féministe, marxiste ou psychanalytique. Dans ce cas, le texte $(\mathrm{T})$ se trouve soumis au sens d'autres textes (TPs) qui constituent la mesure de T, ce qui donnerait TPs/T. Serres note que dans ce cas l'interprète peut aller jusqu'à censurer le texte, et je dirais, à la limite, s'arroger le pouvoir de détruire ce corpus, le nier, le tuer. Ce qui est le cas, dans mon journal de bord, de tous ceux qui, sous prétexte d'esthétique ou de certaines orthodoxies, refusent de voir le film ou, inquisiteurs, seraient prêts à le condamner ou à le brûler. Et je n'y ai pas échappé moimême, à un certain moment.

Notons que Serres donne une définition de l'interprétation fort différente de celle de la praxéologie, qui rejoindrait plutôt en partie son quatrième type, voire un cinquième possible comme nous le verrons plus bas.

\subsubsection{Le quatrième type}

Serres suggère enfin un autre type d'approche: saisir un texte, un corpus comme une réalité nouvelle, inédite, qui aurait sa propre langue à découvrir. En le relisant à partir de lui-même, dans ses propres différences. Comme on découvre ou redécouvre un pays étranger ou quelqu'un de proche. Ce qui doit se rapprocher de L'écoute de Bellet que je n'ai pas lu. Dans ce cas, le texte approché $(\mathrm{T})$ porterait sens par lui-même $(\mathrm{T})$, ce qui donnerait T/T. Ce type d'approche qui respecterait la réalité du corpus, le considérerait en lui-même sans le soumettre, sans le mettre sous quelque chose d'autre $(\mathrm{X})$, ce qui donnerait $\mathrm{X} / \mathrm{T}$. Il est presque totalement absent de mon journal de bord. On y traite peu du film dans sa chair (sauf pour Baroni et Dansereau). On mal-traite le corpus, comme un accusé à son procès qui ne se retrouve pas ou mal dans les représentations partielles et 
partiales de lui-même dans le discours des journalistes, des témoins et des avocats de la couronne, voire de la défense. Au contraire de l'approche du quatrième type qui paraît impartiale, objective.

\subsubsection{Une limite de cette approche du quatrième type}

Ainsi présentée, toute objective, elle occulte cependant le travail du sujet. À la limite, la visée d'objectivité (1) cantonne dans une logique binaire, nie le sujet (S) qui devient (0). Or, un texte, s'il porte sens en lui-même, ne se lit pas lui-même. La découverte de son inédit se fera par un sujet lecteur concret et, par le fait même, dans une pratique économique, selon l'énergie, les ressources, qu'il pourra ou trouvera bon d'y investir. On ne peut s'investir dans tous les corpus, comprendre tous les accusés. Souvent on a du mal à découvrir l'inédit de son propre désir. Je me permets un rapprochement, entre un corpus (texte écrit ou film) dont on devrait s'approcher pour l'investir et le corps du prochain dans l'évangile de Luc (10,25-37). Lorsque l'avocat anxieux demande à Jésus qui est son prochain, une vision binaire de la spiritualité "où le sujet doit s'oublier" amène souvent à répondre par un universel (le prochain = tout humain). La réponse de Jésus est fort différente. De parabole, elle se fait question: "Qui du prêtre, du lévite ou du samaritain s'est fait prochain de l'homme blessé? »Or, l'économie psychique du Samaritain, voyageur riche, mais méprisé en Judée et, par le fait même, vulnérable au voisinage de Jérusalem, l'amène à s'identifier et à se rapprocher du malheureux. Ce qui, paradoxalement, ne semble pas le cas des deux religieux.

\subsubsection{Un cinquième type d'approche?}

Si l'on admet la réalité active du sujet dans le quatrième type et que celleci s'inscrit dans une économie, on peut postuler un autre type d'approche, complémentaire du quatrième. Celui où le sujet a besoin de comprendre le texte pour comprendre et dépasser sa propre conjoncture. Ce qui est la posture de principe de Duhaime que partagent Baroni et Dansereau et que ces derniers illustrent abondamment.

\subsubsection{Réflexions sur ces différentes approches}

Notons tout d'abord que si la typologie les distingue pour bien les définir, il reste qu'en pratique ces différentes approches peuvent se conjuguer dans 
un même discours. Chacune est légitime et on reste libre selon son économie personnelle de choisir l'une ou/et l'autre. Chacune peut aussi fournir du sens valable. Notons particulièrement, dans un cas de figure comme celui de mon journal de bord, que les différents locuteurs ne se limitent pas à approcher le corpus commun (en l'occurrence le film), mais qu'ils l'approchent aussi selon divers types et selon d'autres discours, qu'il s'agisse des corpus écrits (évangiles ou autres) ou de la parole des autres locuteurs. Il s'ensuit que, selon l'économie de chacun, on risque inévitablement de maltraiter les autres corpus ou les autres corps parlants. La violence n'est pas exclusive à l'œuvre/témoignage de Gibson, elle hante sa réception. Comme lui, on reste souvent dans une logique de conformité à un réel (historique ou théorique), dans une logique binaire, où l'un (1) finit toujours par bouffer l'autre (0). Aux environs de la Cité de la paix, de Jérusalem, prêtres et lévites peuvent encore abandonner au fossé le corps blessé d'un autre croyant par pureté rituelle et biblistes et théologiens peuvent aujourd'hui censurer un film en vertu du «de leur savoir théologique».

\subsection{Du monde du signifié à celui du signifiant (présentation schématique ${ }^{13}$ )}

Je voudrais modéliser ce courant de pensée franco-allemand des Bellet, Derrida, Lacan et Saussure, avec en arrière-fond les philosophes allemands de l'interprétation que je n'ai pas lus, mais que j'aperçois au travers de quelques articles de Québécois qui s'en inspirent (A. Fortin, R. Lemieux, F. Nault et G.-R. Saint-Arnaud) et avec lesquels j'ai parfois la joie, trop brève, d'échanger. Des pensées qui sortent de la logique binaire, de celle où le lecteur doit prendre le pouvoir sur le sens (unique) du texte. Des pensées qui creusent, qui évident le texte tout comme le sujet lecteur, qui multiplient les couches d'élaboration de sens chez l'un comme chez l'autre. À deux, on est plusieurs, car chacun apparaît barré par l'autre qu'il est aussi (textelautre; jelautre). La quête de vérité échappe alors à la conquête du sens unique d'un corpus, pour se jouer dans ses innombrables effets de

13. Pour ne pas allonger le texte, mais aussi parce qu'il s'agit d'évoquer un monde dont j'ai l'intuition plus que la maîtrise, cette présentation pourrait bien demeurer schématique. Question intéressante pour la théologie pratique: est-ce que le théologien qui vit au cœur des pratiques doit écrire avec la même maîtrise des sources que le spécialiste dont la pratique exclusive est de s'y consacrer? 
sens qui rendent aussi compte de la richesse de l'histoire de nos libres interprétations.

Cette pensée reste cependant trop souvent confinée en textes théoriques, en des codes linguistiques qui demandent (toujours la question économique qui sous-tend la praxis) un long apprentissage. Il reste à faire passer cette pensée en pratique: peut-être, par exemple, dans le Babel d'agressivité théologique qui surgit autour de La Passion de Gibson?

\subsection{De la nécessité d'une pluralité pour dire DieuE}

Mon carnet de bord relate une bataille de croyants, souvent intellectuels chrétiens ou théologiens, autour d'une autre bataille de croyants, praticiens du verbe (les rabbins et Jésus; les rédacteurs des évangiles qui posent la foi des premières communautés chrétiennes devant celle de la Synagogue). Tout ce monde vit dans un univers de monothéisme où l'on privilégie l'unicité de la vérité; ce qui sera renforcé par l'idée d'empire et qui persistera même à la Réforme. Chaque confession, chaque secte prétend posséder la Vérité. Et la polarisation actuelle reprend souvent cette dynamique. On est loin de Mt 5,17-24.

Dans le Sermon sur la montagne, Jésus associe l'interdiction de tuer, le fait d'être en colère contre son frère, de le traiter de fou. Il précise même que dans ce dernier cas on devra en rendre compte dans le feu de l'enfer. Il commande d'ailleurs d'interrompre le culte dans de telles situations et d'aller se réconcilier avant de le reprendre. Il ne s'agit pas de tomber dans le légalisme, mais la force de l'association du meurtre et de l'injure ou de la condamnation pour hérésie suppose une réflexion éthique qui pourrait s'appliquer aussi bien à la facture du film qu'aux polarisations de sa discussion.

On est loin de cette "parole très importante du Talmud [qui] déclare: "Paroles des uns et des autres, paroles du Dieu vivant." »(Ouaknin 1997, 62) Marc-Alain Ouaknin la cite et commente:

Dieu n'existera comme être infini que si les hommes en font un être vivant à travers l'interprétation [...]. [...] si on en reste à la parole unique [...], alors on crée une idéologie de Dieu [...]. On aboutit tout simplement à la mort de Dieu [...]. La vocation du Talmud — la loi orale des juifs —, c'est de faire éclater la parole unique de la révélation biblique, pour rendre ou garder à Dieu son statut d'infini. (ibid.)

Une théologie pratique qui se démarque d'une logique binaire avec sa violence (dynamique de prise de pouvoir, de réduction du corpus d'un 
discours, voire des personnes qui l'énoncent ou de celles qui en discutent) reste largement à inventer avec ses modes propres d'écriture. Assumer la pluralité d'un corpus comme celle du sujet qui l'aborde et passer de la tyrannie du sens unique au jeu de la pluralité des effets introduiraient au cœur même de l'Église, du religieux et de la culture un œcuménisme dont nous avons grandement besoin.

\section{Une piste d'intervention}

Ce genre de théologie peut commencer en pratique par la mise en place de groupes d'échanges sur le film religieux. Il s'agirait de reprendre la formule du ciné-club. D'un groupe ouvert, où l'on se centre, avant tout, sur le corpus du film en lui-même, quitte à tenir compte, au second plan, de ses références historiques (littéraires ou autres). D'un groupe où l'on cherche moins à statuer sur la valeur du film et sur la vérité de son propos qu'à assumer les diverses lectures des participants dans le respect mutuel.

\section{La prospective}

Quelles seraient les visions anthropologiques, ecclésiales, voire sociales d'une telle démarche? Celles d'un sujet créateur, non soumis à la seule objectivité du film ou des discours d'autorité qui l'entourent. D'un sujet qui accueille l'image dans son propre imaginaire, qui reprend à son compte la possibilité de faire du cinéma et de discuter de sa propre production de sens avec celle d'autres personnes. Celle d'une Église qui s'affranchit de la prétention cléricale d'épuiser la réalité, mais qui n'éclate pas en mille sectes ou solitudes; où l'on continue d'essayer de se comprendre. Celle d'une société démocratique où le sujet résiste au prêt-à-penser de la consommation pour oser sa parole à l'écoute de celle de l'a/Autre.

\section{Références}

Baroni, L. et M. Dansereau (s.d.), «La Passion du Christ: un film intolérable. Ne nous trompons pas de cible», dans les Actes du XVII congrès international de l'Association internationale d'études médico-psychologiques et religieuses, intitulé: Religions et violences? (Strasbourg, 10-15 juillet 2006), à paraître au cours de 2006-2007.

Duhaime, J. (2004), «La Passion du Christ selon Mel Gibson — Quelle fidélité à l'Évangile? » Le Devoir, lundi 24 mars, accessible en ligne à <http://www.ledevoir.com/2004/03/29/50950.html>. 
Gaudreault, A. (1992), "La Passion du Christ: une forme, un genre, un discours ", dans R. Cosandey, A. Gaudreault et T. Gunning, dir., Une invention du diable? Cinéma des premiers temps et religion / An Invention of the Devil? Religion and Early Cinema, Lausanne / Québec, Payot / Presses de l'Université Laval, p. 91-101.

Lemieux, R. (2002), "Le loup dans la bergerie. Théologie et psychanalyse exposées au champ de l'Autre ", Théologiques, 10/2, p. 25-53.

OuAKnin M.-A. (1997), "Lire aux éclats », dans J. Bottéro, M.-A. OuAKNin et J. Moingt, La plus belle histoire de Dieu. Qui est le Dieu de la Bible?, Paris, Seuil, p. 61-63.

Raynauld, I. (1992), «Les scénarios de la Passion selon Pathé (1902-1914) », dans R. Cosandey, A. Gaudreault et T. Gunning, dir., Une invention $d u$ diable? Cinéma des premiers temps et religion / An Invention of the Devil? Religion and Early Cinema, Lausanne / Québec, Payot / Presses de l'Université Laval, p. 131-141.

Serres, M. (1974), Jouvences sur Jules Verne, Paris, Minuit.

Vallin, P. (2004), Note doctrinale sur La Passion du Christ, film de Mel Gibson / dossier spécial de la Commission doctrinale de la Conférence des évêques de France (mars 2004), accessible sur le Web à $<$ http ://www.cef.fr/catho/actus/dossiers/2004/melgibson/vallin.php $>$.

\section{Résumé}

La Passion du Christ de Mel Gibson, témoignage de foi public marqué par la violence, a soulevé bien des passions. Cette contribution tente de rendre compte, dans une perspective praxéologique, du jeu complexe des pratiques et des interactions engagées dans la relation particulière du public et des Églises à un film vu et discuté par des dizaines de millions de personnes à travers le monde. L'auteur, qui s'intéresse tout autant à la médiatisation et à la réception du film qu'à sa facture et à son contenu, rend compte d'une partie des discussions, des appréciations (ecclésiales) et des mises en garde (théologiques) par rapport à ce film. Ce qui ressort de ses observations, c'est la puissance de l'industrie de rêves qu'est le cinéma, mais aussi la variété des manières d'aborder le film, dont certaines sont, tout comme le film, marquées par la violence.

\section{Abstract}

Mel Gibson's The Passion of the Christ, public testimony of faith marked by violence, gave rise to a great deal of passions. This contribution seeks to elucidate, 
in a praxeological perspective, the complex play of practices and interactions involved in the particular relation of the public and the Churches to a movie seen and discussed by millions of people across the world. The author, who is interested as much in the media coverage and reception of the movie as he is in its contents, reports parts of discussions about (ecclesial) appraisals of and (theological) warnings against this movie. What results from his observations is the power of the industry of dreams that cinema is, but also the variety of the ways to approach the movie, a few of which are marked by violence as well. 